\title{
Book review on cognitive engineering and safety organization in air traffic management
}

\author{
Pietro Carlo Cacciabue ${ }^{1}$
}

Published online: 5 October 2018

(c) The Author(s) 2018

This is a very interesting book to read.

It demonstrates why Cognitive Science is necessary for dealing with complex environments involving humans and machines. Air Traffic Management (ATM) is a stereotype such environments, as it presents all the relevant aspects that require the accurate and deep implementation of Cognitive Science concepts. In particular, successful ATM activities imply: (1) collaboration amongst human beings carrying out coordinated work in a control room; (2) cooperation between human beings aiming at the same goal, but acting from distant locations; (3) communication and teamwork activities; and, especially (4) extensive use of automation. Therefore, this book is perfect for marrying the human-machine-interaction (HMI) issues of modern technology and domainspecific requirements.

The authors' grouping is equally appropriate and well balanced for the goals of the book, as it combines an expert scientist in Cognitive Science, with knowledge and familiarity with the air traffic management working environment, and an experienced ATM operator, with deep understanding of HMI in complex domains. Consequently, practical issues and theoretical modelling are equally well balanced throughout the various chapters.

As the authors mention in the Preface, the "book addresses a wide audience of researchers and practitioners" and offers the possibility to scan over the problems and major issues associated to different aspects on ATM management and operations. The "expert" reader will be able to scan the chapters of his/her area of competence, recognising subjects of relevance, and will be able to read about other subject matters and discover what are the essential models, methods and methodologies that are required therein.

Pietro Carlo Cacciabue

p.cacciabue@kingston.ac.uk

1 Kingston University London, Roehampton Vale, Friars Avenue, London SW15 3DW, UK
At the same time, the actual implementation of methods and models is not developed in detail as it requires specific dedicated discussion and review. However, each section and chapter contains a very comprehensive set of references. These enable the reader, in need to go deeper in the subject matter, to know where to find detailed descriptions, guidelines for implementation and strengths and weaknesses of each method and technique.

In essence, this book is certainly valuable for achieving a comprehensive view of the Cognitive Science aspects relevant for ATM and of the methods most commonly applied for problem-solving and assessment of the safety-related topics of the domain.

More in detail, following an important introduction to Cognitive Science and Engineering and its role in assessing designs, controls and processes for effective performances in ATC and ATM, the book is structured in four parts. Each part contains a balanced presentation and discussion between theoretical and practical elements. Part I focuses on the ATM operations and relevant safety-related approaches. The three chapters cover the operations and safety-related elements from the practical and theoretical point of view, discussing the actual operations and the human factors related performances and cognitive aspects. Chapter 3 , in particular, describes extensively the basic four pillars of Safety Management System (SMS) offering various considerations on the sociotechnical and managerial implications of implementing of SMS in ATM, as well as normative regulatory requirements. This part offers a useful overview of the domain from practical and human-related aspects.

Part II concentrates on the Cognitive Science and Cognitive Engineering aspects associated to ATM. Four chapters (Ch. 4-7) cover respectively: Decision Making, Sensemaking, Human Error and Adaptive Practices in ATM. For each cognitive related subject, the major theoretical features and approaches utilised to assess ATM are discussed in some detail. As already mentioned, the actual implementation of a specific method requires a more extensive work 
of knowledge acquisition and review. However, the main processes of implementation are identified and adequately referenced.

Part III is particularly dedicated to the identification and definition of critical elements for non-technical training and implementation of adequate risk management. In particular, Management of abnormal situations (Ch. 8), Workload and complexity (Ch. 9) and new challenges, such as Multimodal information and teamwork management (Ch. 10), are discussed in detail. The essential techniques and associated theoretical framework are reviewed, such as Cognitive Task Analysis, Resilience Engineering approaches and Taskwork/ Teamwork for effective and adaptive management. As for the previous parts, the reader finds in the book the relevant references about each technique where it is possible to find further information and detailed description of means for implementation.

Part IV offers a comprehensive selection of methods and theoretical frames for the implementation of Cognitive Science in ATM (Ch. 11-15). Different models are presented and discussed in some detail, without entering the specific implementation rules and boundary conditions. This part starts by discussing a variety of organisational methods that are well-known and developed over the last 20 years of $\mathrm{R} \& \mathrm{D}$ in human factors (Ch. 11). Chapter 12 concentrates on safety-related issues and incident/accident investigation by scanning the most commonly applied methods and identifying essential issues that need to be revised in retrospective assessment of root causes and identification of genotypes of inappropriate system management. Chapter 13 offers a variety of approaches to couple human behaviour and organisational models to evaluate performances and Chap. 14 concentrates on the Resilience Engineering approach. Finally, Chap. 15 tackles Risk Management for safety assessment, primarily from the point of view of the SMS and effective safety evaluation and development of compliance measures with respect to the norms and standards. Classical methods are shown in association with more recent approaches. As above, the actual selection and implementation of the most appropriate technique to handle the problems of the safety analyst for a Quantitative Risk Assessment requires further reading and study that enable to identify the most adequate approach for the case at hand.

In conclusion, this book offers a comprehensive overview of the relevance and possible contribution of Cognitive Science to the effective design and safety assessment of ATM environment and processes. On its own, it is not sufficient to cover the detail of each specific area of implementation, but it offers a very clear overview of problems, issues and solutions that contribute to consolidating and improving the "Safety" level of the complex, safety critical and continuously evolving domain of Air Traffic Management.

Open Access This article is distributed under the terms of the Creative Commons Attribution 4.0 International License (http://creativeco mmons.org/licenses/by/4.0/), which permits unrestricted use, distribution, and reproduction in any medium, provided you give appropriate credit to the original author(s) and the source, provide a link to the Creative Commons license, and indicate if changes were made. 